différentes instances construisant le débat » (p. 184). Cette conscientisation s'effectue grâce à la réalisation de quelques principes de base qui sont : la conceptualisation des objectifs de l'écoute interprétative par l'apprenant même, la mise en place d'un métalangage adéquat et univoque, et le développement des connaissances rhétoriques en relation avec les catégories de logos, ethos et pathos dans le cadre d'un entraînement stratégique. Une expérience-pilote effectuée par la chercheuse corrobore la validité du modèle intégrant la distinction des séquences relativement stables à leur dynamique rhétorique interne et à la transversalité des stratégies rhétoriques, en munissant l'apprenant des outils permettant une supervision métacognitive de leur activité d'interprétation dans le cadre d'un entraînement stratégique.

Tout porte à croire qu'une telle approche des compétences interprétatives est susceptible de fournir un apport majeur au développement des compétences métacognitive et métadiscursive des apprenants. En effet, la monographie a le mérite d'ouvrir le débat sur les compétences interprétatives en LE, considérées par le CECR comme un axiome et non définies. Il ne nous reste qu'à espérer la publication de résultats confirmant la validité du modèle proposé dans d'autres contextes, d'autant plus qu'il n'est pas exclu que les problèmes indiqués dans l'ouvrage concernent au même titre l'interprétation des discours médiatiques en langue maternelle.

Monika Grabowska ORCID: 0000-0001-7828-0821

Université de Wrocław monika.grabowska@uwr.edu.pl

\title{
L'INCONTOURNABLE EMPRUNT
}

L'emprunt en question(s). Conceptions, réceptions, traitements lexicographiques, sous la direction d'Alicja Kacprzak, Radka Mudrochová et Jean-François Sablayrolles, Éditions Lambert-Lucas, coll. « La Lexicothèque », Limoges 2019, 200 pp., € 24 (paperback), ISBN: 978-2-35935-230-6. https://doi.org/10.19195/0557-2665.67.18

En 2013, en rendant compte d'un ouvrage entièrement consacré au traitement lexicographique de l'emprunt, nous avions souligné que ce phénomène linguistique restait alors au centre de la préoccupation des spécialistes ${ }^{1}$. Une demi-douzaine d'années plus tard, cette constatation n'a rien perdu de son actualité. En témoigne, entre autres, l'activité des Éditions Lambert-Lucas, qui, dans leur collection «La Lexicothèque », dirigée par Christine Jacquet-Pfau et focalisée sur des questions relatives aux unités lexicales

${ }^{1}$ Voir W. Ucherek, compte-rendu de Les dictionnaires et l'emprunt. XVI $-X X I^{e}$ siècle, A. Steuckardt, O. Leclercq, A. Niklas-Salminen et M. Thorel (dir.), Publications de l'Université de Provence, Aix-en-Provence 2011 (Romanica Wratislaviensia LX, 2013, pp. 173-175). 
intéressant plusieurs disciplines (sémantique, lexicologie, lexicographie, dictionnairique), ont récemment publié deux autres études portant sur la problématique de l'emprunt ${ }^{2}$.

Le volume examiné, orienté davantage vers les questions théoriques suscitées par l'emprunt que vers les analyses de cas concrets, comporte dix contributions classées dans ses trois parties qui correspondent chacune à un axe de réflexion différent : examen de la notion même d'emprunt, examen de la réception des emprunts et examen de l'emprunt en tant qu'objet de travaux lexicographiques. L'ouvrage contient aussi une présentation dans laquelle les rédacteurs expliquent le phénomène aux lecteurs et le situent par rapport à d'autres travaux du groupe international de recherche sur la néologie par emprunt ${ }^{3}$ dont ils font partie (pp. 9-15), des informations sur les auteurs (pp. 187-190) et un index des notions (pp. 191196). Si le volume n'a pas de bibliographie commune - ce qui s'explique d'ailleurs tant par la pluralité de ses objectifs que par celle des langues visées (français, italien, polonais, tchèque, néerlandais) —, c'est justement cet index très détaillé qui en renforce la cohérence et en facilite l'interrogation ponctuelle. En outre, nous saluons l'idée de présenter tous les contributeurs; les notes qui leur sont consacrées constituent une autre source de données bibliographiques précieuses.

La première partie, intitulée « Emprunt : concepts connexes et typologie» (pp. 17-86), contient cinq études. Jean-François Sablayrolles ( $«$ Les emprunts face aux xénismes, pérégrinismes, internationalismes, statalismes... », pp. 19-32) évoque quelques termes en rapport avec les mots venus d'ailleurs - en plus de ceux cités dans le titre, il mentionne aussi l'allogénisme et l'étrangisme ${ }^{4}$ - mais focalise plus particulièrement son attention sur le rapport entre les concepts de xénisme et de pérégrinisme. Tout en expliquant les différentes approches de l'emprunt linguistique, l'auteur démontre l'inutilité de la notion de pérégrinisme et précise que la pertinence du concept de xénisme varie selon les situations linguistiques, dans la mesure où il reste opérant avant tout « dans des pays majoritairement monolingues, avec une langue fixée et assez homogène » (p. 31). Le xénisme, compris grosso modo comme « un mot qui désigne un référent considéré comme étranger dans une société donnée » (p. 34), reste au centre de la réflexion de John Humbley (« Requiem pour le xénisme terminologique », pp. 33-46) qui en présente différentes conceptions et signale son caractère non discret, ce qui

${ }^{2}$ Voir Z. Hildenbrand, Les emprunts lexicaux à l'allemand en français contemporain, Éditions Lambert-Lucas, Limoges 2015, et Emprunts néologiques et équivalents autochtones en français, en polonais et en tchèque, Z. Hildenbrand, A. Kacprzak et J.-F. Sablayrolles (dir.), Éditions LambertLucas, Limoges 2016.

${ }^{3}$ Sur les différents procédés d'enrichissement du lexique, dont l'emprunt, considéré comme une matrice lexicale externe, voir J. Pruvost et J.-F. Sablayrolles, Les néologismes, Presses universitaires de France (coll. « Que sais-je ?», no 3674), Paris 2019, pp. 91-115.

${ }^{4}$ On pourrait ajouter à la liste, qui se veut, et pour cause, ouverte, par ex. le terme d'exotisme (la linguistique polonaise considère egzotyzm et ksenizm comme synonymes, et les opinions ne divergent que quant à leur fréquence respective; $c f$. W. Pisarek, «Egzotyzm», [dans :] Encyklopedia języka polskiego, S. Urbańczyk et M. Kucała (dir.), Zakład Narodowy im. Ossolińskich - Wydawnictwo, Wrocław 1999, p. 87 ; B. Walczak, « Kontakty polszczyzny z językami niesłowiańskimi », [dans :] Współczesny język polski, J. Bartmiński (dir.), Wydawnictwo UMCS, Lublin 2014, p. 534 ; A. Markowski, Wykłady z leksykologii, Wydawnictwo Naukowe PWN, Warszawa 2012, p. 129), ou celui d'aliénisme, ce dernier ayant été proposé, il y a environ un demi-siècle, par le (méta)lexicographe russe Valerij Berkov ( $c f$. D. Melnikienè, Le dictionnaire bilingue. Un miroir déformant?, Hermann, Paris 2013, pp. 122-125). 
rend le concept difficile à appliquer dans le traitement automatique du langage, sans pour autant l'exclure des études de néologie, y compris celles de terminologie ${ }^{5}$. En effet, le chercheur est d'avis que si, à cause surtout de la mondialisation, le concept de xénisme devient de plus en plus relatif dans la langue générale ainsi que dans certaines langues de spécialité ( $c f$. secteur des finances), il reste utile dans d'autres ( $c f$. droits nationaux), et aussi, qu'il demeure pertinent en traductologie.

Alicja Kacprzak (« Les linguistes polonais face à l'emprunt. Cadre théorique », pp. 47-55) propose un panorama des travaux sur l'emprunt émanant des linguistes polonais, qui, loin d'aspirer à l'exhaustivité, peut toutefois passer pour représentatif. Après avoir mentionné la notion de mélange de langues, présente notamment dans les travaux des philologues allemands du XIX ${ }^{\mathrm{e}}$ siècle, l'auteure évoque les travaux polonais s'inscrivant dans la linguistique générale, qui abordent l'emprunt dans le cadre plus large des contacts entre les langues (Jan Niecisław Baudouin de Courtenay, Jerzy Kuryłowicz, Witold Cienkowski), proposent des classements (Adam Weinsberg ${ }^{6}$ ) et prennent position par rapport à la thèse de la puissance (innée) des langues (Adam Weinsberg, Władysław Miodunka); dans un troisième temps, elle passe aux travaux centrés sur l'emprunt en polonais et sur ses typologies (Aleksander Brückner, Stanisław Szober, Halina Kurkowska et Stanisław Skorupka). Cet article est en quelque sorte prolongé par le suivant, « Les linguistes polonais face à l'emprunt au français : quelques approches méthodologiques » (pp. 57-72). En effet, contrairement à ce que suggère le titre, ses auteurs, Anna Bobińska et Andrzej Napieralski, ne se limitent pas aux emprunts français, mais décrivent brièvement certaines approches qu'ont du phénomène d'autres spécialistes polonais (les auteurs reconnaissent en toute modestie qu'il s'agit d'un échantillon de travaux ${ }^{7}$ ), telles les approches historique et culturelle (Edward Szmańda), sémantique (Ryszard Tokarski), étymologique (Witold Cienkowski) ou relative aux types d'assimilation (Władysław Cyran) ${ }^{8}$,

5 À ce propos, consulter J. Humbley, La néologie terminologique, Éditions Lambert-Lucas, Limoges 2018.

${ }^{6}$ En relatant les idées de Weinsberg, exposées dans son livre Językoznawstwo ogólne (PWN, Warszawa 1983, pp. 77-82), l'auteure mentionne les « langues de la branche balkanique », ce qui trahit un problème d'équivalence terminologique. En effet, Weinsberg se sert du terme liga językowa (littéralement 'ligue de langues'), censé rendre le terme allemand Sprachbund dont le correspondant français le plus répandu semble être union de langues, employé notamment par Patrick Sériot (cf. par ex. Le nom des langues en Europe centrale, orientale et balkanique, P. Sériot (dir.), Éditions Lambert-Lucas, Limoges 2019, passim) ; le terme aire de convergence linguistique est également en usage. Ainsi, bałkańska liga językowa est à rendre par union de langues balkaniques.

${ }^{7}$ Parmi les études laissées (momentanément ?) à l'écart, citons par ex. celle de Barbara Reczkowa, à orientation dialectologique, qui porte sur la présence des mots d'origine française dans les patois polonais (Wyrazy pochodzenia francuskiego $w$ gwarach polskich, Zakład Narodowy im. Ossolińskich - Wydawnictwo, Wrocław 1982) ou celle de Bronisława Ligara, focalisée sur l'idiolecte d'un grand écrivain polonais du XIX ${ }^{\mathrm{e}}$ siècle, Zygmunt Krasiński (1812-1859), et plus précisément, sur la présence des gallicismes dans sa correspondance (Galicyzmy leksykalne w listach Zygmunta Krasińskiego na tle wplywów francuskich w polszczyźnie XIX wieku, Uniwersytet Jagielloński, Kraków 1987).

${ }^{8}$ On peut toutefois se demander pourquoi, en exposant les pensées de Halina Rybicka, les auteurs se basent uniquement sur son bref article de 1967 (« W sprawie wyrazów obcych w języku polskim », Poradnik Językowy 2, pp. 93-98) et ne mentionnent pas son livre Losy wyrazów obcych w języku polskim (PWN, Warszawa 1976). 
pour focaliser leur attention sur la vision globale de l'emprunt présente dans de nombreuses études de Bogdan Walczak. Ils proposent ensuite une synthèse des observations de Walczak au sujet de l'impact du français sur le polonais et terminent sur la présentation de la méthodologie adoptée par la romaniste Anna Bochnakowa dans la rédaction de son dictionnaire des emprunts du français ${ }^{9}$.

Alors que les linguistes polonais contemporains se servent avant tout du terme zapożyczenie ('emprunt'; son synonyme, de moins en moins utilisé, est pożyczka), bien ancré dans l'usage, en tchèque, il existe trois termes principaux, výpưjčka, přjejimka et přejaté slovo. Comme le démontre Radka Mudruchová («À la recherche de la typologie et de la définition de l'emprunt dans le milieu linguistique tchèque », pp. 73-86), même si certains linguistes en privilégient ou en évitent un ( $c f$. une certaine prédilection de František Čermak pour výpưjčka, terme critiqué par Diana Svobodová), cette disproportion d'usage se laissant également remarquer dans des dictionnaires de langue, d'autres, dont l'auteure, les perçoivent comme synonymes et estiment qu'on peut les substituer l'un à l'autre. Dans son étude, Mudruchová mentionne également quelques autres termes liés à l'emprunt, tel barbarismus, xenismus, pseudoprejimka ('faux emprunt') et internacionalismus, passe en revue plusieurs classements d'emprunts, en mettant en avance les critères retenus (avec, en tête, le degré d'adaptation formelle), et dénonce le statut marginal des études néologiques dans le contexte tchèque.

La deuxième partie du volume («Réception des emprunts », pp. 87-131) renferme trois contributions centrées sur l'analyse des attitudes des locuteurs de trois langues emprunteuses - italien, néerlandais et tchèque - face à l'emprunt, et sur les causes de ces attitudes. Giovanni Tallarico (« Emprunts et gallicismes dans la langue italienne : trois siècles de postures idéologiques ", pp. 89-101) touche à la question du nombre de gallicismes (les estimations vont d'environ 1500 à plus de 4000 items si on inclut près de 3000 emprunts adaptés) pour abandonner l'approche quantitative au profit d'une perspective socio-culturelle. Après avoir sommairement retracé l'histoire de l'influence du français sur l'italien, le chercheur se concentre sur les trois derniers siècles et parle tour à tour de la gallomanie du XVIII ${ }^{\mathrm{e}}$ siècle, qui a entraîné l'essor des gallicismes, et de ses opposants, les tenants des idées universalistes se faisant de moins en moins nombreux ; de la montée, dès la fin de ce siècle, du « purisme de matrice nationaliste » (p. 93) qui, malgré l'existence des voix opposées à la xénophobie linguistique, a marqué le siècle suivant si bien qu'il n'était alors plus possible de dissocier le débat sur les emprunts d'une prise de position idéologique, en particulier anti ou profrançaise ; enfin, de la réalité de la première moitié du $\mathrm{XX}^{\mathrm{e}}$ siècle où le purisme, lié au mythe de l'italianité, a trouvé un nouvel allié dans le fascisme, de sorte qu'il a fallu attendre la fin du régime pour que l'opposition aux emprunts faiblisse. L'auteur conclut que tout au long de la période discutée, l'emprunt reste « un enjeu à la fois esthétique, linguistique et politique » (p. 99).

L'étude de Matthieu Pierens (« L'emprunt en néerlandais : réalité et perception », pp. 103-120) s'ouvre sur une brève présentation de la langue néerlandaise ${ }^{10}$, pour laquelle

${ }^{9}$ Wyrazy francuskiego pochodzenia we wspótczesnym języku polskim, A. Bochnakowa (dir.), Wydawnictwo Uniwersytetu Jagiellońskiego, Kraków 2012 ; voir le compte-rendu de Stefan Kaufman paru dans Romanica Wratislaviensia LX, 2013, pp. 171-173.

${ }^{10}$ Il est difficile de savoir sur quelle base l'auteur affirme que « le néerlandais est parlé par environ 29 millions de locuteurs aux Pays-Bas et en Belgique (23 millions aux Pays-Bas et 6 millions en Belgique) » (p. 103). En effet, selon l'office néerlandais des statistiques (CBS), le nombre total 
il existe deux standards linguistiques différents, celui des Pays-Bas et de la Belgique, suivie d'une synthèse sur les emprunts dans une optique quantitative, les emprunts au français, au latin, à l'anglais et à l'allemand faisant l'objet d'un développement. Ensuite, l'auteur adopte une perspective sociolinguistique en s'interrogeant sur la capacité des locuteurs à identifier les emprunts comme tels et sur leur attitude envers ces mots, et signale l'existence d'un mouvement puriste qui, jusqu'au XIX ${ }^{\mathrm{e}}$ siècle, s'oppose au français, puis à l'allemand et, à partir du milieu du $\mathrm{XX}^{\mathrm{e}}$ siècle, à l'anglais. Concernant la théorisation linguistique, Pierens souligne que la diversité régionale et l'écart entre les deux standards du néerlandais orientent les spécialistes plutôt vers la piste sociolinguistique et historique, encore qu'ils aient élaboré un classement original de type ternaire (mots indigènes, bâtards et étrangers), s'éloignant ainsi du modèle binaire allemand (mots indigènes vs exogènes).

Jan Lazar (« La perception du phénomène d'emprunt dans le milieu linguistique tchèque et ses possibles classements », pp. 121-131) affirme que parmi les différentes typologies tchèques des emprunts ( $c f$. article de Radka Mudruchová, pp. 77-80), c'est celle qui renvoie à l'origine des mots qui semble être la plus souvent retenue, et il se concentre par la suite sur les emprunts que le tchèque doit à trois langues majeures, le français, l'anglais et l'allemand. La même grille d'analyse est rigoureusement appliquée : historique des relations entre les populations parlant une langue pourvoyeuse d'emprunts et les tchécophones, revue des champs sémantiques auxquels appartiennent les gallicismes, anglicismes ou germanismes, et examen des mots appartenant à chacune de ces trois catégories sous l'angle de leur degré d'intégration à la langue tchèque. L'auteur parvient à la conclusion qu'au niveau de l'adaptation phonétique, ce sont les germanismes qui s'assimilent le plus facilement, à la différence des gallicismes ; les anglicismes, quant à eux, gardent souvent leur prononciation d'origine. En outre, ces derniers posent le plus de difficultés lors de l'adaptation morphologique, à cause notamment de l'absence de catégorie de genre en anglais. Enfin, Lazar constate qu'actuellement, le français n'enrichit pratiquement plus le vocabulaire tchèque alors que l'allemand continue de le faire grâce à la proximité géographique des deux zones linguistiques.

La troisième et dernière partie («Emprunts, lexicographes et veille néologique », pp. 133-185) n'est composée que de deux contributions. Anna Bochnakowa (« Les lexicographes polonais du XIX ${ }^{\mathrm{e}}$ au $\mathrm{XXI}^{\mathrm{e}}$ siècle face à l'emprunt », pp. 135-143) propose une synthèse des pratiques lexicographiques en matière de mots d'origine étrangère basée sur un corpus sélectif de cinq dictionnaires généraux et d'une dizaine de dictionnaires de mots étrangers, ces derniers étant bien ancrés dans la tradition lexicographique polonaise. L'auteure affirme que les emprunts sont largement présents dans les nomenclatures des dictionnaires de langue, dont les rédacteurs ne semblent pas avoir cédé à d'éventuelles tendances puristes, et que le nombre élevé des dictionnaires spécialisés qui répertorient ces mots s'explique par un souci pédagogique, pour finalement inviter à entreprendre des études diachroniques plus approfondies, centrées sur l'évolution des emprunts figurant dans les ouvrages de ce type.

d'habitants des Pays-Bas reste inférieur à 17,5 millions ( $c f .<$ https://www.cbs.nl/en-gb/visualisaties/ population-counter $>$ ). Cette information converge avec les données fournies par Wikipedia, d'après lesquelles 23 millions est le nombre total de néerlandophones. 
Dans un ample texte très bien documenté, Emmanuel Cartier («Emprunts en français actue $^{11}$ : étude linguistique et statistique à partir de la plateforme Néoveille », pp. 145185) expose en détails plusieurs jalons théoriques de sa recherche (il cerne la notion d'emprunt tout en la comparant à des notions connexes, y compris celle de néologisme) et les points essentiels de sa méthodologie, liée notamment à l'utilisation de la plateforme Néoveille (caractérisation du corpus, manière de valider les emprunts, leur description linguistique et socio-pragmatique, suivi méticuleux de leur évolution). Il en ressort qu'il importe de prendre en compte « trois moments saillants de la vie des néologismes : l'émergence, l'éventuelle diffusion et l'éventuelle lexicalisation » (p. 170). En guise d'illustration, Cartier expose les résultats de l'analyse des emprunts en français repérés et validés dans Néoveille dans les années 2015-2017.

L'ensemble de ce volume collectif, qui contient des études de qualité, se lit agréablement et fait honneur à ses contributeurs. Outre les études focalisées sur une langue romane, en l'occurrence le français et l'italien, son intérêt scientifique réside d'une part dans la diffusion des idées linguistiques, tant récentes que plus anciennes, formulées dans des langues moins connues (néerlandais, polonais, tchèque), et d'autre part, dans la propagation du savoir relatif à l'influence du français sur le lexique de ces langues.

Witold Ucherek

ORCID: 0000-0002-7954-7206

Université de Wroctaw witold.ucherek@uwr.edu.pl

${ }^{11}$ Dans la version du titre figurant dans la table des matières, actuel est remplacé par contemporain, et dans la « Présentation » (p. 14), le titre est formulé d'une manière nettement différente. 